
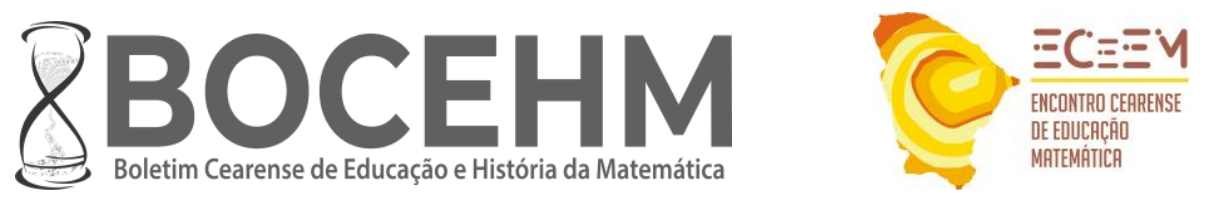

ISSN: $2447-8504$

DOI: $10.30938 /$ bocehm.v8i23.5065

(®⿻) $\odot$

\title{
ANÁLISE DE RESOLUÇÕES DE PROBLEMAS DE PARTILHA À LUZ DA TEORIA DA OBJETIVAÇÃO
}

\section{ANALYSIS OF RESOLUTIONS TO SHARING PROBLEMS IN LIGHT OF OBJECTIVATION THEORY}

\author{
Anailde Felix Marques ${ }^{1}$; Marcelo Lins Muniz de Melo Santos²; Jadilson Ramos de \\ Almeida $^{3}$
}

\begin{abstract}
RESUMO
Este artigo surgiu durante a disciplina de Didática da Álgebra, na qual realizamos leituras e discussões sobre vários artigos, desde a abordagem do contexto histórico, passando pelos documentos oficiais que regulamentam o ensino de Álgebra no Brasil. Por fim, discutimos as diferentes perspectivas do pensamento algébrico sob o ponto de vista de diferentes teóricos, analisadas também em pesquisas recentes. A partir disso, os autores desta investigação ficaram responsáveis por analisar as estratégias adotadas por três estudantes, dos anos finais do Ensino Fundamental, ao resolverem problemas de partilha. Essa análise foi feita à luz das camadas do pensamento algébrico, o factual, o contextual e o padrão, descritos por Radford (2009). A metodologia deste trabalho teve um caráter qualitativo, cujos dados foram coletados por Almeida (2016) durante seu doutorado na cidade de Recife - PE. Em sua tese, o pesquisador entrevistou oito alunos de diferentes séries dos anos finais do Ensino Fundamental, dos quais disponibilizou as anotações e as entrevistas de cinco deles. No entanto, neste trabalho, iremos apresentar a análise de apenas três desses estudantes dentro das perspectivas estabelecidas. Nesse sentido, os dados foram analisados observando-se traços ou indícios pertinentes ao pensamento algébrico nas camadas de generalidade propostas, a partir das quais pode-se ter uma concepção dos níveis de desenvolvimento algébrico desses estudantes. Como limitação a este trabalho, durante a coleta de
\end{abstract}

${ }^{1}$ Licenciada em Matemática pela Universidade Federal de Campina Grande (UFCG). Mestranda no Programa de Pós-graduação em Educação Matemática e Tecnológica pela Universidade Federal de Pernambuco (EDUMATEC - UFPE). Recife, Pernambuco, Brasil. Endereço para correspondência: Avenida Acadêmico Hélio Ramos s/n, Cidade Universitária, Recife, Pernambuco, CEP: 50670901. E-mail: anailde.felix@ufpe.br.

(iD) ORCID iD: https://orcid.org/0000-0001-5517-1024.

${ }^{2}$ Licenciado em Matemática pela Universidade Federal de Pernambuco (UFPE). Mestrando no Programa de Pós-graduação em Educação Matemática e Tecnológica pela Universidade Federal de Pernambuco (EDUMATEC - UFPE). Recife, Pernambuco, Brasil. Endereço para correspondência: Avenida Acadêmico Hélio Ramos s/n, Cidade Universitária, Recife, Pernambuco, CEP: 50670901. E-mail: marcelo.msantos@ufpe.br.

ORCID iD: https://orcid.org/0000-0002-5016-6258.

${ }^{3}$ Doutor em Ensino das Ciências e Matemática pela Universidade Federal Rural de Pernambuco (UFRPE). Professor do Curso de Licenciatura em Matemática e do Programa de Pós-graduação em Ensino das Ciências (PPGEC) pela Universidade Federal Rural de Pernambuco. Também é professor no Programa de Programa de Pós-graduação em Educação Matemática e Tecnológica (EDUMATEC-UFPE). Recife, Pernambuco, Brasil. Endereço para correspondência: Rua Dom Manoel de Medeiros, s/n, Dois irmãos, Recife, Pernambuco, Brasil, CEP: 52171900. E-mail: jadilson.almeida@ufpe.br.

(iD) ORCID iD: https://orcid.org/0000-0003-3707-4807. 
dados, Almeida (2016) não realizou gravações de áudio ou vídeo durante a sequência de atividades propostas aos estudantes. Em vista disso, não foi possível analisar os dados com profundidade na perspectiva do pensamento algébrico, uma vez que, segundo a Teoria da Objetivação, o gestual e o corporal são aspectos fundamentais dessa teoria e possivelmente elementos deixaram de ser capturados nesse processo.

Palavras-chave: Pensamento algébrico; Educação Matemática; Álgebra escolar.

\begin{abstract}
This article comes from the module Didactics of Algebra, which we carry out readings and discussions on various articles, from the approach of the historical context, through the official documents that regulate the teaching of algebra in Brazil. Finally, we discuss the different perspectives of algebraic thinking from the point of view of different theorists, also analyzed in recent research. From this, the authors were responsible for analyzing the strategies adopted by three students, from middle school, when they were solving problems of sharing. This analysis was made in the light of the elements that characterize algebraic thinking, such as factual, contextual and standard, described by Radford (2009). Therefore, we can affirm that the methodology of this work had a qualitative character. The data were collected by Almeida (2016) during his doctorate, in the city of Recife - PE. In this study, this researcher interviewed 8 students from different grades of middle school, of which the author provided the notes and interviews of only 5 of these students from different grades. However, in this work, we will present the analysis of three students within the presented perspectives. With that, these data were analyzed observing relevant traits or evidence of algebraic thinking in the proposed layers of generality, from which one can have a conception of the levels of al gebraic development of these students. As a limitation of this work, during data collection, Almeida (2016) did not record audio and video during the sequence of activities proposed to students. Thus, we could not do the analysis of the perspective of algebraic thinking, according to the Theory of Objectification, because the gestural and corporal are fundamental aspects of this theory and possibly elements are no longer captured in this process.
\end{abstract}

Keywords: Algebraic thinking; Mathematical Education; School algebra. 


\section{Introdução}

Esta pesquisa é oriunda da disciplina de Didática da Álgebra, cujos encontros foram realizados remotamente devido às medidas de distanciamento social decretadas pelas autoridades da área de saúde, a fim de evitar a propagação do vírus da covid-19. Inicialmente, tivemos contato com uma diversidade de artigos que abordam o ensino da Álgebra, começando pela análise do contexto histórico dessa disciplina no Brasil. Discutimos os pressupostos dos Parâmetros Curriculares Nacionais até chegar aos que estão em vigor, atualmente denominados Base Nacional Curricular Comum (BNCC). Em seguida, debatemos as bases epistemológicas do ensino da Álgebra, bem como as diferentes vertentes do pensamento algébrico.

Almeida e Câmara (2017) expõem a pertinência do pensamento algébrico, respaldando-se na assertiva de que não existe concordância na literatura sobre o que seja esse pensamento, mas apenas a importância de se pensar algebricamente. Com esse fundamento, os autores caracterizaram concepções sob diferentes prismas do pensamento algébrico, baseados em três teóricos: Rômulo Lins, James Kaput e Luis Radford. Nesse mesmo viés, acrescentam a importância do pensamento algébrico, buscando mobilizá-lo no sentido de auxiliar os professores da educação básica a planejar suas aulas.

Apesar de Radford (2006) e Lins (1992) dialogarem no mesmo sentido, Radford nos indica que, quando estamos pensando algebricamente e à medida que manipulamos analiticamente objetos algébricos desconhecidos, estes podem estar associados por meio de elementos semióticos, como os sinais. Assim, entendemos que Radford (2006) discursa na mesma compreensão de Vygotsky (1997), no que diz respeito à linguagem. Isso porque, para Vygotsky, segundo apontam Fernandes e Healy (2017), a linguagem é um conceito amplo que abrange, além da função comunicativa, a função de organização e de desenvolvimento dos processos de pensamento. Entendemos, portanto, que essa perspectiva semiótica é a que melhor irá fundamentar nossas análises neste trabalho.

A opção pelo pensamento algébrico na perspectiva da Teoria da Objetivação (TO) fundamenta-se no favorecimento de situações que tornam viáveis as modificações em sujeitos éticos e reflexivos, contribuindo para a formação de opinião destes em relação a problemas da sociedade e consequente desenvolvimento pessoal (GOMES; NORONHA, 2020). Com isso, tendo por base as leituras e discussões que cercam a TO, realizadas durante a disciplina, assim como os estudos de Almeida (2016) e Almeida e Câmara 
(2017, 2018), surgiu a questão: como seriam realizadas as análises dos dados fornecidos por Almeida e Câmara (2018), com base nos três elementos da camada de generalização (factual, contextual e padrão) levantadas por Radford (2009)?

Ante o exposto, o objetivo deste artigo será analisar o raciocínio desenvolvido por estudantes dos anos finais do $\mathrm{EF}$, ao resolver problemas de partilha à luz das camadas do pensamento algébrico (factual, contextual e padrão) descritos por Radford (2009). Como objetivos específicos, analisaremos as resoluções apresentadas e as entrevistas realizadas com os sujeitos participantes, com base nas três camadas de generalização à luz da perspectiva do pensamento algébrico. Os dados da pesquisa foram coletados por Almeida (2016), durante o seu doutorado, observando-se os diferentes níveis do pensamento algébrico (ALMEIDA; CÂMARA, 2018), em escolas da cidade de Recife-PE, tendo sido, posteriormente, publicados no artigo: Desenvolvimento do Pensamento Algébrico: proposição de um modelo para os problemas de partilha, na revista Zetetiké em 2018.

\section{Referencial teórico}

Neste tópico, descrevemos alguns aspectos fundamentais para o entendimento da TO, como a ancoragem da teoria. Em destaque, daremos ênfase nas camadas da generalidade do pensamento algébrico, tendo em vista serem os elementos fundamentais para a nossa análise e discussão.

Segundo Radford (2020), a TO desfruta da influência de Marx e Hegel no materialismo dialético e Vygotsky, na perspectiva histórico-cultural. Indo mais além, o arcabouço teórico desses pesquisadores percorre a influência da cultura no desenvolvimento do ser humano. Com um olhar epistêmico, percebemos as aproximações empiristas, construtivistas e epistemológicas sobre o sujeito-objeto, o que complementaria as perspectivas anteriores. Portanto, concebe-se o ensino-aprendizagem como um único processo, e por essa circunstância, enriquecem-se as reflexões sobre o saber e o ser. Nesse sentido, Radford (2020, p. 16) acrescenta que se faz necessário redefinir " [...] os conceitos de saber e aprender de uma maneira consistente com a abordagem histórico-cultural" (tradução nossa ${ }^{4}$ ).

\footnotetext{
4 "los conceptos de saber y aprendizaje de manera coherente con una aproximación histórico-cultural" (RADFORD, 2020, p. 16).
} 
Concisamente, para a TO, a cultura é o elemento chave, no qual se viabiliza o processo de aperfeiçoamento da sociedade como um todo; e, em especial, o de ensinoaprendizagem, observando-se as características que rodeiam o sujeito, com a intencionalidade voltada para a matemática. A teoria também possui inquietações sobre:

[...] o quê, o porquê, como e quem aprende, ou seja, atenta para os aspectos ontológicos (essência dos objetos de conhecimento) e epistemológicos (como os objetos de conhecimento são aprendidos). Nessa Teoria, os conceitos de saber, conhecimento, aluno, professor, ensino e aprendizagem são (re)significados, a partir da perspectiva histórico-cultural de Vygotsky (GOMES; NORONHA, 2020, p. 137).

A teoria contribui para novas reflexões sobre ações e pensamentos, elaboradas por demandas históricas e culturais, que favorecem a construção de sujeitos que possuem discernimento para se posicionar frente a situações (RADFORD, 2009, 2014, 2020; VERGEL, 2015). Do ponto de vista da TO, o pensamento consiste em uma prática social, cultural e multimodal, ou seja, auxilia na observação de situações por ângulos diferentes (GOMES; NORONHA, 2020). Exemplificando: o pensamento algébrico não se reduz apenas à aplicação de equações e incógnitas; o aluno pode realizar movimentos circulares com um lápis no ar, e isso é visto como uma mobilização do pensamento algébrico. Daí a importância de filmar, gravar áudios e tirar fotos, pois, momentos como esses, muitas vezes não são capturados no decorrer da aula pelo professor ou pesquisador. Conclui-se, então, que a generalidade do pensamento algébrico pode acontecer pela linguagem verbal (oral e escrita), ou não-verbal, por exemplo, o gestual, o corporal (RADFORD, 2009).

Segundo afirma Radford (2009), existem três camadas distintas atribuídas ao pensamento algébrico denominadas por: factual, contextual e padrão. Assim, como fizeram Fernandes e Healy (2017), para distinguir e descrever cada uma das camadas do pensamento algébrico, iremos utilizar a descrição e os resultados encontrados nos estudos de Radford (2009).

A partir disso, uma atividade foi desenvolvida com os estudantes do $8^{\circ}$ e $9^{\circ}$ ano. A princípio, a finalidade era visitar os conteúdos e observar o progresso desses alunos. A turma foi inicialmente dividida em grupos com três estudantes cada, aos quais se propôs a atividade que consistia na abordagem da generalização de padrões mediante uma sequência, como na Figura 1. 
Figura 1: Atividade da generalização de padrões

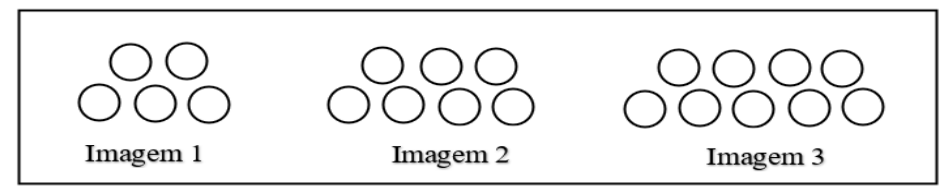

Fonte: Adaptado de Radford (2009)

A problematização constituiu-se em duas grandes etapas. Na primeira, os estudantes continuaram desenhando a figura 1, tendo por objetivo representar as imagens 4 e 5. Em seguida, procederam à busca pelo número de círculos correspondentes às imagens 10 e 100. Na segunda etapa da atividade, eles escreveram uma mensagem para um estudante de outra turma, diferente da deles, demonstrando como encontrar o número de círculos de uma figura, seja qual for; desenvolvendo, assim, uma fórmula para representar o número de círculos (RADFORD, 2009).

A seguir, detalhamos por subtópicos cada uma das características descritas por Radford $(2009,2014)$ e outros pesquisadores que corroboram seu pensamento, tal como Vergel $(2015,2016)$.

\section{Pensamento Algébrico Factual}

No primeiro momento, os estudantes optaram por determinar o número de círculos das imagens 1, 2 e 3, observando, na sequência, o aumento gradativo em cada uma delas. Isto significa que, eles identificaram a adição de dois círculos a cada imagem, a título de exemplo: imagem 1 - 5 círculos, imagem $2-7$ círculos, imagem 3 - 9 círculos. Conseguiram compreender a regularidade entre o número da imagem e o número de círculos correspondente (RADFORD, 2009), realizando a divisão das imagens por meio de linhas.

A partir daí, sugeriram assertivas tais como: "o tempo todo vai ter um no ar", fazendo referência a que sempre vai existir um círculo a mais na linha inferior. Essa camada do pensamento envolve a indeterminação, números desconhecidos (incógnitas) de forma implícita. Ademais, gestos, movimentos e palavras são elementos integrantes da semiótica dos estudantes (RADFORD, 2009). No entanto, Radford alerta que reconhecer a regularidade não é uma condição capaz de garantir a percepção do número de círculos das imagens 100, 1.000 e 10.000, tendo em mente que isso não é um argumento suficiente para justificar a compreensão da generalidade.

Almeida e Câmara (2017) descrevem que: 
Por exemplo, o aluno é capaz de perceber que para calcular o número total de círculos da figura 10, basta somar 1 ao 10 para encontrar o número de círculos da linha superior, que nesse caso é igual a 11, e somar 2 ao 10 para encontrar o número de círculos da linha inferior, que é igual a 12. Logo, o total de círculos da figura 10 é igual à soma dos círculos da linha superior com o total de círculos da linha inferior, ou seja, 23 círculos. Para calcular o número de círculos da figura 100 o estudante utiliza a mesma estrutura, ou seja, $101+$ 102, que é igual a 203 círculos (p. 49).

Com isso, observamos a recorrência do termo "fórmula-em-ação" de Vergnaud, que Radford (2009) diz ter pego emprestado. No entanto, realizou algumas ressalvas como: “uma 'fórmula' dessa forma concreta do pensamento algébrico pode ser entendida como um predicado concretizado por uma variável implícita: indeterminação não chega ao nível do discurso" (RADFORD, 2009, p. 9, tradução nossa ${ }^{5}$ ).

Assim, a indeterminação é vista como uma lacuna que precisa ser preenchida por termos específicos. Por fim, o autor afirma que o pensamento algébrico factual “[...] não é uma forma simples de reflexão matemática. Pelo contrário, baseia-se em mecanismos altamente evoluídos de percepção e uma coordenação rítmica sofisticada de gestos, palavras e símbolos” (Ibid., p. 9, tradução nossa ${ }^{6}$ ).

\section{Pensamento Algébrico Contextual}

Nessa camada do pensamento, observamos um nível de aprofundamento maior comparando-o com o anterior, justamente porque os estudantes são convidados a realizar a segunda parte da atividade. Devemos aqui lembrar que os alunos tinham que escrever uma mensagem para um outro, descrevendo como calcular o número de círculos de uma imagem qualquer.

De acordo com Radford (2009), a tarefa solicita que os estudantes deixem de lado as figuras particulares e busquem o novo objeto - a figura geral. A indeterminação assume outra perspectiva nessa camada, sendo considerada como elemento chave na linguagem. Posto isso, apresentamos a resolução de um estudante do $9^{\circ}$ ano e observamos alguns

\footnotetext{
5 "A 'formula' of this concrete form of algebraic thinking can better be understood as an embodied predicate with a tacit variable: indeterminacy does not reach the level of discourse" (RADFORD, 2009, p. 9).

6 " [...] is not a simple form of mathematical reflection. On the contrary, it rests on highly evolved mechanisms of perception and a sophisticated rhythmic coordination of gestures, words, and symbols" (RADFORD, 2009, p. 9).
} 
aspectos que serão descritos mais adiante. A atividade consistia em adicionar dois círculos por imagem sequencialmente.

Essa resolução facilita a distinção entre o pensamento factual e o pensamento contextual. Consiste na substituição da ação e da percepção por palavras que possuem uma representatividade superior ou inferior. Essa atribuição linguística denomina-se dêiticos que, em outras palavras, são utilizados para descrever, de modo contextual, objetos em espaços. Para Radford (2009, p. 10),

No entanto, embora seja diferente do pensamento algébrico factual, ambos em termos da forma como a indeterminação é tratada e os meios semióticos que os alunos pensem, a nova forma de pensamento algébrico ainda é contextual e "perspectiva" no sentido de que é com base em uma maneira particular de considerar algo (tradução nossa ${ }^{7}$ ).

\section{Pensamento Algébrico Padrão}

Nessa camada do pensamento, os mecanismos são altamente evoluídos e consequentemente difíceis de serem mobilizados pelos estudantes. Inclusive, Radford (2009), em algumas oportunidades, atribuiu-lhes outro nome: pensamento algébrico simbólico, considerando que, anteriormente, os alunos poderiam utilizar diversos recursos da semiótica, tais como gestos, movimentos e elementos dêiticos. Entretanto, nesta última do pensamento, eles estabelecem conexões com as fórmulas algébricas na base alfanumérica, em outras palavras, a linguagem algébrica simbólica. Reconhecem que as fórmulas alfanuméricas contam histórias sobre os fenômenos vivenciados (ALMEIDA; CÂMARA, 2017).

Nessa etapa da tarefa, a finalidade era que os estudantes conseguissem escrever a fórmula: $(\mathrm{n}+1)(\mathrm{n}+2)$ para representar o número de círculos da imagem. Radford (2009) descreve que um dos grupos tentou realizar essa atividade por tentativa e erro, modificando os números na medida do necessário, produzindo fórmulas que não são algébricas, por exemplo: $\mathrm{n} \times 2+4$ ou n x $2+2$. Segundo o autor, funcionou para alguns casos, mas para outros não.

\footnotetext{
7 "However, although different from factual algebraic thinking both in terms of the way indeterminacy is handled and the semiotic means which the students think, the new form of algebraic thinking is still contextual and "perspectival" in that it is based on a particular way of regarding something" (RADFORD, 2009, p. 10)
} 
Em relação a isso, Almeida e Câmara (2017, p. 52) afirmam que

Em um nível mais avançado do pensamento algébrico simbólico, o aluno é capaz de simplificar essa fórmula, chegando a uma que não seja uma descrição espacial da figura, mas uma síntese da relação existente entre o número da figura e o número de círculos. Temos, a seguir, uma fórmula simplificada para representar o número de círculos da figura $n: 2 n+3$.

Pela nossa observação de Almeida e Câmara (2017), na perspectiva do pensamento algébrico nessa camada, interpretamos que esta possui subníveis do desenvolvimento, além de que elementos fundamentais foram deixados de lado, como superior e inferior, justamente porque eles não são partes integrantes da abstração. Ao mesmo tempo, Radford (2009) descreve a importância do caminho trilhado pelos estudantes desde o factual e o contextual até o padrão, sempre buscando o desenvolvimento do pensar algebricamente.

\section{Metodologia}

Durante a disciplina Tópicos de Matemática: Didática da Álgebra do Programa de Pós-Graduação em Ensino de Ciências da UFRPE, tivemos abordagens que nos conduziram para reflexões/discussões sobre as três perspectivas do pensamento algébrico dos autores Rômulo Lins, James Kaput e Luis Radford. A turma foi então dividida em três grupos e cada um deles ficou responsável por analisar, sob a perspectiva de um dos autores citados acima, o material coletado por Almeida (2016).

Sendo assim, este artigo baseia-se em uma abordagem qualitativa, principalmente pela ênfase em dados descritivos, vistos como favoráveis para a interpretação, tal como descrevem Bogdan e Biklen (1994). Portanto, este trabalho consiste em analisar as anotações e as entrevistas realizadas com estudantes dos anos finais do Ensino Fundamental, sob o ponto de vista dos três elementos da camada de generalização segundo Radford (2009): factual, contextual e padrão. Os dados desse estudo foram coletados por Almeida (2016) durante o seu doutorado na cidade de Recife - PE.

A pesquisa foi realizada com oito alunos de séries diferentes. No entanto, o pesquisador disponibilizou as anotações e as entrevistas de apenas três, também de séries diferentes, cujos nomes fictícios foram listados no decorrer do texto.

Depois da atividade realizada com os estudantes sujeitos da pesquisa, foi possível observar algumas das estratégias utilizadas ao resolverem problemas de partilha. No 
entanto, isso não foi suficiente para satisfazer o objetivo do estudo, tendo em vista que as características do pensamento algébrico basicamente são mentais, e que “[...] não podemos ficar apenas na análise dos registros escritos, pois eles talvez não nos revelem com clareza o que o aluno estaria pensando no momento em que estava resolvendo o problema" (ALMEIDA; CÂMARA, 2018, p. 552). Portanto, alguns dias após a aplicação da atividade, foi realizada com os alunos uma entrevista para explicitação, a fim de auxiliar na identificação de elementos característicos do pensamento algébrico. A seguir, apresentamos as anotações e as entrevistas dos participantes que foram disponibilizadas para esta investigação.

\section{Resultados e discussão}

As questões abaixo, cedidas por Almeida (2016), foram analisadas à luz da Teoria da Objetivação de Radford (2009), apresentando e discutindo os traços pertinentes ao pensamento algébrico a partir da resolução dos problemas e das entrevistas realizadas.

Q6b. Sílvia, Pedro e Carlos querem dividir 70 figurinhas entre eles, de modo que Sílvia receba 30 figurinhas a menos que Pedro e a metade da quantidade de figurinhas de Carlos. Quantas figurinhas cada um vai receber?

Figura 3 - Resposta da aluna Carol $\left(9^{\circ}\right.$ ano) à Q6b

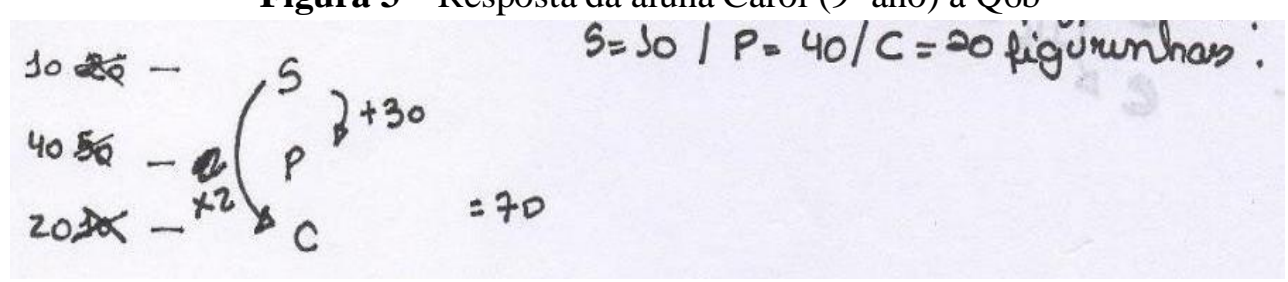

Fonte: Almeida e Câmara (2018)

\section{Extrato da entrevista com Carol}

P: Ok Carol. E esse segundo? Me explica esses esquemas que você fez.

Carol: Isso aqui é Sílvia, Pedro e Carlos (apontando para S, P e C), e isso aqui é o total de figurinhas (apontando para o " $=70 "$ ).

P: Por isso você colocou igual a 70?

Carol: Foi. Então, de Sílvia para Pedro, Sílvia receberia 30 figurinhas a menos que Pedro, então eu sabia que, vamos supor, se Sílvia tem X, X mais 30 seria o valor de Pedro.

P: Por que ela recebe 30 a menos que Pedro, então Pedro receberia 30 a mais que ela?

Carol: Isso. Então eu fui fazendo isso também, e a metade de Carlos, então se ela tinha metade, então X vezes 2 seria igual a Carlos.

P: Por que Carlos seria o dobro de Sílvia, é isso? 
Carol: Isso.

P: Certo. Carol, agora me explica como foi que você chegou nesses valores aqui?

Carol: Também por estimativa.

P: Primeiro você tentou com 20 , foi isso?

Carol: Foi.

P: E quando somou não bateu com o resultado?

Carol: É. Deu um número a mais, deu 80.

P: Por isso você tentou com 10 ?

Carol: Isso.

P: Vê, você tenta com 20 , e deu 80 , é isso que você está falando?

Carol: Isso.

P: Então, você diminuiu a quantidade para 10 para ver se dá 70 ?

Carol: Foi.

P: Não poderia aumentar, no caso? Você não tentaria com um número maior que esse?

Carol: Não. Se fosse maior, sairia ainda mais dos 70.

P: Entendi. Então você diminuiu porque você sabe que tem que ser um número menor para dar 70, por que o valor que você encontrou primeiro foi maior?

Carol: Isso. E nos outros também foi a mesma coisa.

Um dos primeiros passos efetuados por Carol consistiu na representação das personagens pelas iniciais dos nomes: S (Sílvia), P (Pedro) e C (Carlos) e, na sequência, a atribuição de valores: $\mathrm{S}=20, \mathrm{P}=50$ e $\mathrm{C}=10$. No entanto, ao efetuar a soma dos números de figurinhas que cada um irá receber, Carol percebeu um acréscimo no quantitativo, o que não poderia ser feito, tendo em vista que o total das três personagens era de 70 figurinhas. Desse modo, ela optou por 10, para tentar encontrar o número correto de cada um.

Ao mesmo tempo, Carol revela que as tentativas não foram realizadas aleatoriamente devido a um aspecto que relata na entrevista: "Se fosse maior, sairia ainda mais dos 70" (ALMEIDA; CÂMARA, 2018, p. 556). Esse trecho torna nítida a percepção de Carol sobre o problema. Em relação aos outros aspectos - (x2) para representar que o número de figurinhas de Carlos é duas vezes maior que o de Sílvia; (+30) para descrever que Pedro possui o número de figurinhas de Sílvia mais 30 - são descritos nas anotações feitas a fim de elucidar o problema,

Nota-se, então, que Carol mobilizou alguns aspectos do pensamento algébrico, considerando que ela objetivou a regularidade da situação proposta no problema ao estabelecer o número de figurinhas de cada personagem. A aluna considerou também a 
indeterminação dos elementos e os números desconhecidos, como se viu em sua explicação: “... se Sílvia tem X, o valor de Pedro será X mais 30”. A partir dessa análise, revelou-se relações e significados atribuídos por ela aos objetos algébricos e que, portanto, há indícios de pensamento factual descrita por Radford (2009).

Q1b. Frederico, Rogério e Lúcia têm, juntos, 55 revistas em quadrinhos. Rogério tem o dobro de revistas de Frederico e Lúcia tem 15 revistas a mais que Frederico. Quantas revistas tem cada um?

Figura 4 - Resposta da aluna Polly ( $9^{\circ}$ ano) à Q1b

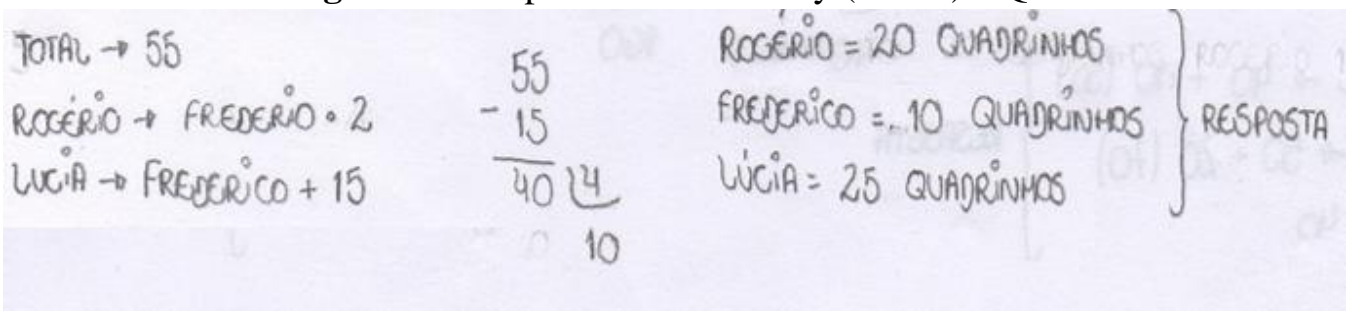

Fonte: Almeida e Câmara (2018)

\section{Extrato da entrevista com Polly}

P: Olá Polly, tudo bom? A ideia é você explicar como chegou nas respostas dos problemas. Por exemplo, esse primeiro, como foi que você fez?

Polly: Na verdade, eu comecei pela quinta questão, foi quando eu percebi a lógica das questões, que eram todas as mesmas. Então eu voltei para o primeiro, e fiz a questão. Eu peguei e subtraí a quantidade que tinha a mais e depois eu dividi pela quantidade de pessoas que tinha.

P: Mas nesse caso tinha três pessoas!

Polly: Isso. Mas tinha uma pessoa que tinha o dobro, então, tinha que ser uma quantidade que conseguisse, tipo, tinha três pessoas, mas uma pessoa tinha o dobro de revistas de Frederico. Rogério tinha o dobro de Frederico, então, teoricamente seriam quatro quantidades.

P: Por isso você dividiu por 4 ?

Polly: Por isso dividi por 4.

P: Então, você tira os 15, que é o que Lúcia tem a mais?

Polly: Lúcia tem a mais que Frederico.

P: Então você tirou esse 15 para ficar em quantidades iguais para cada um?

Polly: Sim.

P: Nesse caso você percebe que Rogério tem o dobro de Frederico, então Rogério teria duas quantidades, é isso?

Polly: Sim.

P: E por isso você, em vez de dividir por 3, dividiu por 4 ?

Polly: Sim.

P: E quando você dividiu por 4 você achou a quantidade de quem?

Polly: Eu achei a quantidade de Frederico, que é a pessoa que Rogério tem o dobro e Lúcia tem mais 15. Ele seria meio que o único que teria uma quantidade certinha. Então, Frederico teria 10, Rogério teria duas vezes Frederico, e Lúcia teria Frederico mais 15, como Frederico é 10, seria 20 e 25. 
P: Então, você pega o Frederico como base, que você achou a quantidade, que seria 10. E Rogério teria o dobro, que seria 20 , é isso?

Polly: Sim. E Lúcia teria Frederico mais 15, que fica 25.

Logo no início da entrevista com Polly, um trecho em especial chama a atenção: "Na verdade, eu comecei pela quinta questão, foi quando eu percebi a lógica das questões, que eram todas as mesmas" (ALMEIDA, 2016, p. 143). A aluna facilmente percebeu a regularidade das sequências propostas a sua turma e, a partir disso, utilizou esse artifício a seu favor para solucionar todos os problemas.

Ainda observamos, no decorrer do diálogo, que ela descreve aspectos importantes, como a realização da subtração para retirar uma parte que tinha a mais e, com o resto da operação, efetuou uma divisão por 4. Portanto, o argumento utilizado é que uma das personagens tem o dobro da outra. Esses elementos do discurso de Polly apresentam indícios do pensamento algébrico contextual (RADFORD, 2009), principalmente pela “[...] mudança para representar a relação covariacional [...]" (SILVA; FAJARDO, 2020, p. 33), usando a palavra dobro ao invés de escrever (x2), como Carol o fez no problema anterior. Assim, temos a mudança com a inclusão do termo dobro, ou seja, um dêitico. Segundo Radford (2009), a ênfase, nessa camada, recai sobre palavras que favorecem a representatividade: dobro, triplo, superior ou inferior.

Q3b. Três times de basquete participam da final do campeonato fazendo, juntos, 240 pontos. $O$ time $B$ fez o dobro de pontos do time A e o time $C$ fez 40 pontos a mais que o time B. Quantos pontos fez cada time?

Figura 5 - Resposta da aluna Júlia ( $7^{\circ}$ ano $)$ à Q3b

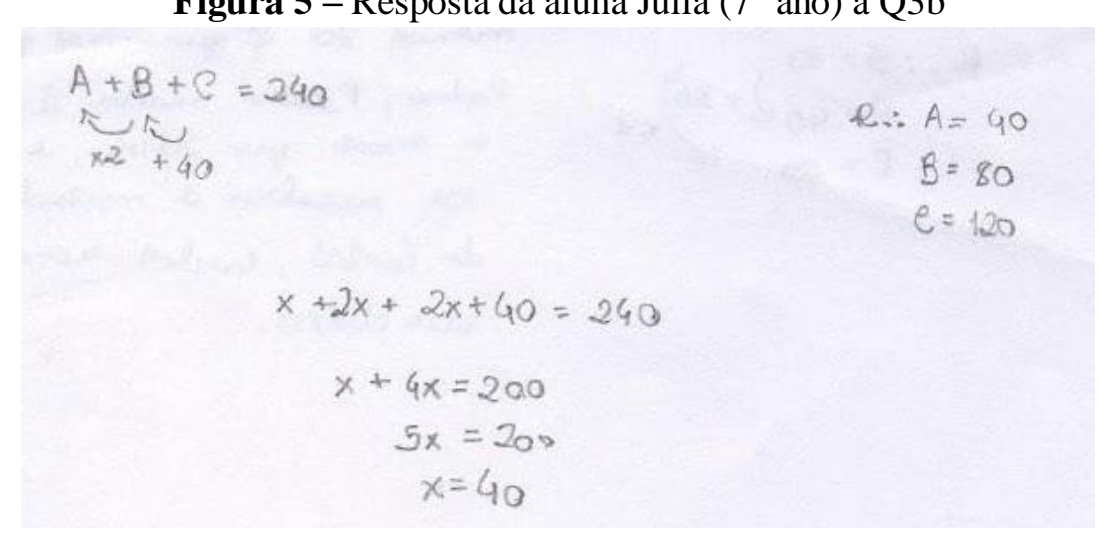

Fonte: Almeida e Câmara (2018)

\section{Extrato da entrevista com Júlia}

P: Ok. Agora Júlia, me explica como você fez esse terceiro. 
Júlia: (Depois de ler o problema) O time B fez o dobro de pontos do time A (apontando, no modelo inicial, para $(\overleftarrow{x 2})$ ), e o time $\mathrm{C}$ fez 40 pontos a mais que o time B (apontando, no modelo inicial, para $(\overleftarrow{+40})$ ).

P: Certo.

Júlia: Então, o A vai ser X, mais $2 X$, que seria $X$ vezes 2, que é o B, porque o B é o dobro do A, mais $2 X$, que seria o B, mais 40, e essa equação seria o C (chama $2 X+40$ de equação), que é igual a 240.

P: Certo.

Júlia: Então, X mais 2X mais 2X mais 40 é igual 240. Então, eu diminuí 40 dos dois lados, e ficou X mais $4 X$, porque eu juntei os $2 X$ com os $2 X$, que fica igual a 200 . E depois eu somei $X$, que ficou $5 X$ igual a 200. Então, $X$ é igual a 40, porque eu dividi 5 dos dois lados.

P: Certo. E depois, você fez como?

Júlia: Então, o time A ficou com 40, o time B ficou com o dobro do time A, que é 80, e o time C ficou com mais 40 vezes 2 , que seria 120 .

P: Certo.

A partir da resolução de Júlia, percebemos a organização do problema em duas partes: primeiro, quando opera com as letras para representar os times de basquete $(\mathrm{A}+$ $\mathrm{B}+\mathrm{C}=240)$. Em seguida, indo no mesmo raciocínio, utiliza setas para designar: “[...] $\left(\overleftarrow{x_{2}}\right)$, indo de B para A, para indicar que o time B fez o dobro de pontos do time A, e $(\overleftarrow{+40})$ indo de C para B, sinalizando que o time C fez 40 pontos a mais que o time B" (ALMEIDA; CÂMARA, 2018, p. 561). Na segunda parte, Júlia transforma os dados do problema em uma equação polinomial do $1^{\circ}$ grau, na qual $\mathrm{A}$ corresponde ao $\mathrm{X}$, o B é equivalente ao $2 \mathrm{x}$, justamente porque é o dobro de $\mathrm{A}$, enquanto o $\mathrm{C}$ é igual ao valor de $\mathrm{B}$ mais 40, ou seja, $2 x+40$. Daí, temos que: $\mathbf{x}+\mathbf{2 x}+\mathbf{2 x}+\mathbf{4 0}=\mathbf{2 4 0}$.

Por meio de todos os dados, descritos na resolução e na entrevista, notamos o pensamento de Júlia em um nível avançado, levando em consideração que estamos analisando as camadas do pensamento algébrico mobilizado pela estudante que, por exemplo, conseguiu objetivar a regularidade no número de pontos que cada time de basquete fez com o total. Compreendendo que os dêiticos fazem parte do processo de oralidade para explicar a resolução, observamos que ela escreveu uma fórmula seguindo a conexão com os dados disponibilizados pelos problemas e a base alfanumérica. $\mathrm{Na}$ resolução desse problema, Júlia mobilizou as três camadas do pensamento algébrico descrito por Radford (2009), desde a factual, a contextual até a padrão.

\section{Considerações finais}

A partir das análises realizadas, podemos concluir que a aluna Carol conseguiu desenvolver a primeira camada do pensamento algébrico, ou seja, a factual. Assim sendo, 
concordamos com a perspectiva de Almeida e Câmara (2018), quando afirmam que essa aluna possui um nível incipiente de pensamento algébrico. Analisando os dados da entrevista de Polly, foi observado que ela desenvolveu os dois primeiros níveis de algebrização, ou seja, factual e contextual. Sendo assim, concordando com a visão de Almeida e Câmara (2018), consideramos que ela possui um nível intermediário de pensamento algébrico. Por fim, Júlia mobilizou os três níveis de pensamento algébrico (factual, contextual e padrão). Isso demonstra que há uma consolidação no nível do pensamento algébrico dessa aluna (ALMEIDA; CÂMARA, 2018).

Ao longo da coleta de dados, Almeida (2016) não realizou gravações de áudio ou de vídeo durante a sequência de atividades proposta aos estudantes. Portanto, essa foi uma adversidade encontrada ao tentarmos analisar os mesmos dados na perspectiva do pensamento algébrico segundo a TO. Isso porque o gestual e o corporal são aspectos fundamentais dessa teoria e, diante desse entrave, possivelmente elementos significativos deixaram de ser capturados nesse processo. Apesar de o fato ter sido uma limitação ao nosso trabalho, compreendemos, ao mesmo tempo, que esses aspectos não eram fundamentais para o pesquisador na época.

\section{Referências}

ALMEIDA, J. R. de. Níveis de desenvolvimento do pensamento algébrico: um modelo para os problemas de partilha de quantidade. 2016, $200 \mathrm{f}$. Tese (Doutorado em Ensino de Ciências e Matemática) - Universidade Federal Rural de Pernambuco, Recife.

ALMEIDA, J. R. de.; CÂMARA, M. dos S. Desenvolvimento do Pensamento Algébrico: proposição de um modelo para os problemas de partilha. Zetetiké: Campinas - SP, v. 26, n. 3, p. 546-568, 2018.

ALMEIDA, J. R. de.; CÂMARA, M. dos S. Pensamento algébrico: em busca de uma definição. RPEM: Campo Mourão - PR, v. 6, n. 10, p. 34-60, 2017. Disponível em: http://rpem.unespar.edu.br/index.php/rpem/article/download/1124/972. Acesso em: 26 fev. 2021.

BOGDAN, R. C.; BIKLEN, S. K. Investigação Qualitativa em Educação: uma introdução à teoria e aos métodos. Porto: Porto Editora, 1994.

FERNANDES, S. H. A. A.; HEALY, L. A emergência do pensamento algébrico nas atividades de aprendizes surdos. Ciência \& Educação: Bauru - SP, v. 22, n. 1, p. 237$252,2016$. 
GOMES, L. P. da S.; NORONHA, C. A. Caracterização do pensamento algébrico na perspectiva da Teoria da Objetivação. In: GOBARA, S. T.; RADFORD, L. (org.). Teoria da Objetivação: Fundamentos e aplicações para o ensino e aprendizagem de ciências e matemática. São Paulo: Editora Livraria da Física, 2020.

LINS, R. C. A framework for understanding what algebraic thinking is. Tese (Doctor of Philosophy) - School of Education, University of Nothingam, Nothingam, UK: 1992.

RADFORD, L. Algebraic thinking and the generalization of patterns: a semiotic perspective. In: North America Conference of the International Group of Psychology of Mathematics Education - PME. Bergen University College. v. 1, 2006.

RADFORD, L. Signs, gestures, meanings: Algebraic thinking from a cultural semiotic perspective. Lyon, França, 2009.

RADFORD, L. The progressive development of early-embodied algebraic thinking. Mathematics Education Research Journal, Dordrecht, v. 26, n. 2, p. 257-277, 2014.

RADFORD, L. Un recorrido a través de la teoría de la objetivación. In: GOBARA, S. T.; RADFORD, L. (org.). Teoria da Objetivação: Fundamentos e aplicações para o ensino e aprendizagem de ciências e matemática. São Paulo: Editora Livraria da Física, 2020.

SILVA, J. G. da.; FAJARDO, R. O pensamento algébrico segundo a teoria da objetivação: análise de episódios de trabalho conjunto no $5^{\circ}$ ano do Ensino

Fundamental. Educação Matemática em Revista: Rio Grande do Sul - RS, v. 1, n. 21, p. 23-34, 2020. Disponível em:

http://sbem.iuri0094.hospedagemdesites.ws/revista/index.php/EMR-

RS/article/view/2244/1713. Acesso em 15 jan. 2021.

VERGEL, R. ¿Cómo emerge el pensamiento algebraico? el caso del pensamiento algebraico factual. Uno: Revista de Didáctica de las Matemáticas. n. 68, p. 9-17, 2015.

VERGEL, R. El gesto el ritmo en la generalización de patrones. Uno: Revista de Didáctica de las Matemáticas. n. 73, p. 23-30, 2016.

VYGOTSKY, L. S. Obras escogidas V: fundamentos da defectología. Madrid: Visor, 1997.

VYGOTSKY, L. S. The genetic roots of thought and speech. In: HANFMANN, E.; VAKAR, G. (Ed.). Thought and language. Cambridge: M.I.T. Press, 1997. p. 73-102.

Recebido em: 06 / 03 / 2021

Aprovado em: 16 / 04 / 2021 